\title{
Motor-cognitive approach and aerobic training: a synergism for rehabilitative intervention in Parkinson's disease
}

\author{
Davide Ferrazzoli ${ }^{*} \ddagger, 1$ (i) , Paola Ortelli ${ }^{\ddagger}, 1$ Alberto Cucca ${ }^{2,3}$, Leila Bakdounes², Margherita \\ Canesi $^{\S, 1}$ \& Daniele Volpe 2 \\ ${ }^{1}$ Fresco Parkinson Center, Department of Parkinson's disease, Movement Disorders \& Brain Injury Rehabilitation, \\ 'Moriggia-Pelascini' Hospital - Gravedona ed Uniti, Como, 22015, Italy \\ ${ }^{2}$ Fresco Parkinson Center, Villa Margherita, S. Stefano Riabilitazione, Vicenza, 36057, Italy \\ ${ }^{3}$ The Marlene \& Paolo Fresco Institute for Parkinson's \& Movement Disorders, Department of Neurology, NYU School of Medicine \\ New York, NY 10017, USA \\ *Author for correspondence: Tel.: +39 33186 43163; Fax: +39 03448 5896; davideferrazzoli@gmail.com \\ $\ddagger$ These authors contributed equally to this work \\ $\S$ These authors contributed equally to this work
}

\section{Practice points}

- Parkinson's disease (PD) is not a mere 'movement disorder,' but rather it is a complex motor behavior disease responsible for a tremendous social and economic impact.

- The optimal management of PD should involve integrated, multidisciplinary approaches combining both pharmacotherapy and non-pharmacological interventions, such as rehabilitation.

- Bottom-up and top-down cognitive coping strategies and adaptive techniques are useful for achieving motor benefits in patients with PD.

- The aerobic exercise may promote neural rearrangements and improve cognition in patients with PD.

- Combining a 'goal-based', motor-cognitive practice with aerobic training seems to provide sustained clinical benefits rather than conventional physical therapy in patients with PD.

- Neuroplastic changes probably drive the clinical rehabilitation-induced benefits in patients with PD.

- Future studies should identify optimal parameters of intensity, frequency and duration of rehabilitation in patients with PD.

Parkinson's disease (PD) results in a complex deterioration of motor behavior. Effective pharmacological or surgical treatments addressing the whole spectrum of both motor and cognitive symptoms are lacking. The cumulative functional impairment may have devastating socio-economic consequences on both patients and caregivers. Comprehensive models of care based on multidisciplinary approaches may succeed in better addressing the overall complexity of PD. Neurorehabilitation is a highly promising nonpharmacological intervention for managing PD. The scientific rationale beyond rehabilitation and its practical applicability remain to be established. In the present perspective, we aim to discuss the current evidence supporting integrated motor-cognitive and aerobic rehabilitation approaches for patients with PD while suggesting a practical framework to optimize this intervention in the next future.

First draft submitted: 13 October 2019; Accepted for publication: 10 December 2019; Published online: 10 February 2020

Keywords: aerobic exercise $\bullet$ motor-cognitive rehabilitation $\bullet$ neuroplasticity $\bullet$ Parkinson's disease $\bullet$ quality of life

Parkinson's disease (PD) is the second most common neurodegenerative disorder. In the near future, the prevalence of $\mathrm{PD}$ is expected to exceed that of any other known neurological disorder, including Alzheimer's disease. Indeed, according to recent epidemiologic projections, the number of individuals affected by PD is poised for exponential growth [1].

The dopaminergic neuronal loss in the substantia nigra pars compacta is universally regarded as the pathological hallmark of PD [2]. The resulting altered connectivity in cortico-basal ganglia networks is believed to be involved 
in the pathophysiology of the cardinal motor symptoms of the disease, including bradykinesia, rigidity and resting tremor [3].

The clinical spectrum of PD, however, encompasses several non-motor features, which are known to be significant determinants of patients' quality of life (QoL) [4]. These include mood changes, anxiety, apathy, fatigue, sleep disturbances, chronic pain, gastrointestinal abnormalities and bladder dysfunction - among others.

Although originally described in terms of motor symptoms, it is generally acknowledged that nowadays PD should no longer be regarded as a mere 'movement disorder,' but rather as a complex motor behavior disease. Consolidated experimental evidence demonstrates how basal ganglia nuclei receive multimodal sensorimotor, cognitive and emotional information from converging cortical pathways and generate a 'compressed' and highly integrated output message to the frontal cortex, where the selection of a proper motor behavior is finally elaborated [5,6]. Notably, this highly integrated computational process encodes for any internally generated motor behavior: from the simplest movement of a single joint, to the most complex motor sequence involving the entire body in order to express emotional contents in a given cognitive setting [7].

This broad physiological complexity should indeed be appreciated by clinical scientists in order to understand the complex phenomenology of PD. Along the disease course, the neurodegenerative process spreads to other, nondopaminergic neural systems, such as the cholinergic and the noradrenergic pathways [8,9]. At this stage, patients usually develop cognitive, motivational and more complex 'axial' disturbances affecting gait, balance and posture, whose responsiveness to dopamine replacement therapy (DRT) is almost always suboptimal, if not disappointing. Not uncommonly, these conditions may overlap in the same patient, thus exerting an overall detrimental impact on QoL as well as social and emotional well-being [10-12].

Indeed, while in early PD DRT is usually effective in improving self-perceived QoL by addressing the dominant motor features of the disease [13,14], its effectiveness in the medium-advanced stages tends to decrement. Furthermore, the potential onset of DRT-related side effects, such as motor fluctuations, dyskinesia, painful dystonia, dopamine dysregulation syndrome and impulsive-compulsive disorders conspire, over time, to reduce the overall tolerability of pharmacological therapies [15].

Given the multi-layered complexity of PD, it is generally acknowledged that its optimal management should involve multidisciplinary approaches combining both pharmacotherapy and non-pharmacological interventions $[16,17]$. Among the latter, growing evidence supports neurorehabilitation as an effective complementary treatment for the management of PD [18-26]. Neurorehabilitation could be defined as the set of clinical and carer interventions aimed at recovery from nervous system damage (due to acquired injuries or to degenerative diseases) by reducing or compensating for the functional disturbances by using the patients' individual neuroplastic resources.

In the past years, the complex interplay between basal ganglia and cortical-cerebellar networks in the modulation of both cognitive-motivational (non-motor) and motor aspects of action [5,27-31] has been increasingly considered in the development of novel integrated rehabilitative approaches (see Figure 1). Furthermore, emerging evidence from basic science and clinical studies suggested the potential for add-on aerobic exercise to boost brain plasticity mainly through long-term potentiation (LTP) phenomena [32].

In the current perspective, we aim to discuss the potential for integrated motor-cognitive rehabilitation practices and aerobic exercise to harness greater and more sustained clinical benefits than any uni-dimensional rehabilitative approach alone. Potential ways to combine these two methodologies within routine rehabilitation protocols will be finally discussed.

\section{Motor-cognitive intervention in PD: scientific rationale \& clinical evidence}

A number of different rehabilitative protocols designed for PD were previously reported in the clinical literature [20,21]. The vast majority of them focused on improving basic motor aspects of PD [33-36]. Overall, results from these interventions were mixed and somewhat conflicting. Further, these protocols did not expressly investigate the influence of cognitive processes in modulating patients' final motor behavior [5,27-31].

However, mounting evidence suggests that the broad degenerative process underlying PD may manifest with a complex clinical phenomenology involving not only primary motor aspects of action, but also cognitive, emotional and motivational drivers $[5,26,29,30,37]$. This huge neural networks disruption could explain why PD motor signs and symptoms may be preceded or accompanied by a wide range of neuropsychiatric features (such as anxiety, apathy, depression, fatigue and psychosis) [38], whose potential impact on functional outcomes has been described in the rehabilitation field [39,40]. In the last years, the importance of engaging subject's cognition in order to achieve greater motor benefits began to be increasingly considered [37]. More specifically, the impairment of basal 


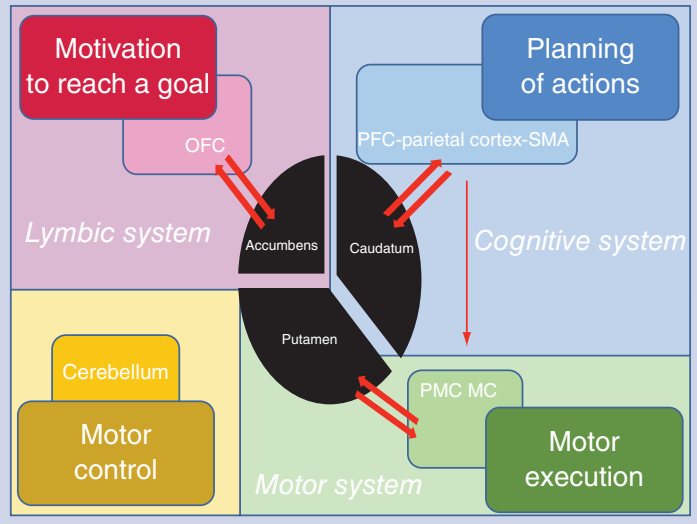

Figure 1. The role of the basal ganglia-cortico-cerebellar networks in motor behavior: implications for rehabilitation.

Schematically, the striatum can be divided into three principal nuclei with different functions: the nucleus accumbens plays a crucial role in decision-making and in reward-based learning; the nucleus caudatum is fundamental for motor learning and for action planning; finally, the putamen has a pivotal role for scheduling and executing habitual-automatic motor skills. These nuclei are strictly interconnected with limbic, associative and sensorimotor cortical areas, respectively. From a functional point of view, these corticostriatal structures are central to learn and express each single motor behavior: the motivation to move for reaching a goal is driven by the accumbens-OFC network; the network linking the caudatum with PFC, parietal cortex and SMA represents the neural system for action planning; motor execution is finally provided by the putamen-SMA-PMC-MC network. The cerebellum monitors the motor performance, contributes to error correction of the 'ongoing' action and generates a continuous signal for maintaining the congruency of the motor behavior. Considering this functional complexity, in a rehabilitative perspective, the treatment of a neurological disorder leading to the disruption of motor behavior (such as Parkinson's disease) has to be designed considering all the motor, motivational and cognitive aspects underlying the dysfunction. OFC: Orbitofrontal cortex; PFC: Prefrontal cortex; PMC: Premotor cortex; MC: Motor cortex; SMA: Supplementary motor area.

ganglia-cortical-cerebellar networks in PD has been involved in: i) an aberrant expression of habitual-automatic, goal-based actions [41-44], ii) the dysregulation of reward-based [45-47] and error-based learning processes [48,49], and iii) an impaired switching from automatic to voluntary and goal-based actions [50-53]. The aforementioned mechanisms are believed to underline the pathophysiology of bradykinesia [54], walking problems - including freezing of gait [55], postural abnormalities and balance disturbances with falls [56-58], writing and manual dexterity problems [59], speech [60] and swallowing deficits [61] in these patients.

It therefore follows that, from a cognitive and motor learning perspective, rehabilitation in PD should aim to foster the re-acquisition of lost habitual, goal-based motor behavior [37]. To achieve this result, clinicians may take advantage of multidisciplinary approaches [26] including physiotherapy, occupational therapy [62], speech and swallowing therapies [63].

Although both the implicit learning and executive control may be affected in PD [64-68], it has been shown that $\mathrm{PD}$ patients remain capable to apply bottom-up and top-down cognitive coping strategies and benefit from adaptive techniques. These include explicit cues, such as verbal instructions, and implicit cues, such as environmental sounds or visual signals $[37,69-75]$.

It is believed that cues allow patients for engaging the volitional-executive control of movements, thus bypassing dysfunctional sensorimotor-habitual networks, while reinforcing the cortical mechanisms involved in motor drive [76-78]. While the explicit cues likely exploit executive functions mediated by the caudatum-prefrontal cortex network, implicit cues are thought to act through entrainment and attention focusing via the cerebellum-prefrontal cortex network $[37,79,80]$.

An additional rehabilitative strategy involves the use of feedback-based learning. The underlying neural pathway seems to be found in the striatum-prefrontal cortex network [81]. Feedback-based motor learning involves the volitional, constant modulation of the ongoing motor behaviors based on internal and/or external signals that are strictly action-specific [82,83]. 
The overall effects of adaptive techniques (external cues, internal cues and feedback-based motor learning) may translate into clinical improvements on mobility, balance, gait, posture - among others [70-72,75,84].

The fact that PD patients may effectively apply to cognitive strategies in order to cope with their motor disability suggests that rehabilitative interventions engaging cognition may indeed harness motor learning schemata leading to broad clinical, motor and functional benefits [32]. As a case in point, many robotic-mechanical devices adopted in the field of neurological rehabilitation engage cognition to achieve motor benefits. Treadmill training represents the prototype among the tools used in this motor-cognitive perspective. The main goals of treadmill training are improving gait and enhancing patients' physiological reserve. Training parameters such as speed and workload can be individualized for each subject, while the intensity and challenge of the training can be dynamically adjusted overtime. While using this device, kinetic signals provided by the sliding belt allow patients for focusing their attention to the active control of gait. As such, treadmill training may exert a normalizing effect on spatiotemporal gait parameters, thus improving gait rhythmicity and reducing gait variability in patients with PD, especially when associated with cues, feedback and music cueing [85-87]. Worthy of interest in this field is the use of virtual reality (VR), a computerized simulation that allows patients to interact with a virtual environment through multiple sensorial modalities [88,89], thus stimulating both motor and cognitive processes, simultaneously [90]. VR provides augmented feedback about performance and may enable individualized repetitive practice of motor function. The use of VR provides patients with benefits in the short and long-term period [91,92].

Another effective cognitive rehabilitative technique is motor imagery (MI). This is based on a conscious access with the intention to move. A large body of evidence suggests that imagined and executed actions share the same neural structures, recruiting overlapping regions [90,93,94]. Consistently, it has been demonstrated that MI may improve both motor performance and motor learning processes [94]. In the same way, Action Observation Therapy (AOT) represents a further, interesting and effective cognitive instrument for motor rehabilitation in PD [94]. AOT is based on the observation and imitation of specific motor actions for facilitating the motor learning processes, probably through the activation of the so-called 'mirror neurons' system [95,96]. A recent study demonstrated that this technique is effective in treating freezing of gait and that benefits may be sustained over a 4-weeks follow-up period [96]. The cognitively-mediated effects of this modality of rehabilitation were recently supported by imaging studies reporting an increased recruitment of frontoparietal areas in patients undergoing AOT [97]. Over the past years, motor-cognitive training showed a great efficacy in ameliorating a lot of goal-based, automatic motor behaviors, thus corroborating the valuable role of these strategies for the management of parkinsonian patients [37].

In this scenario, dual task training is a prime example of motor-cognitive interplay and a highly appealing technique to be implemented in the management of PD [98].

In $\mathrm{PD}$, gait function tends to shift from a healthy, semi-automatic pattern to a maladaptive profile where attention-based strategies are inappropriately used to maintain locomotion. These compensatory strategies are not optimized for natural walking and when a second cognitive task is applied, the resulting attention shift may decrease walking speed and smoothness, ultimately leading to gait disruption and falls [99,100].

While in PD the abrupt administration of dual tasks can exert disruptive effects on walking, compelling evidence also suggests that motor strategies based on repetitive dual task training and dual task reinforcement may improve the cognitive reserve of these patients, thus ameliorating their gait function in clinical and ecological settings [101,102].

Based on previous reports from stroke literature [103], Yang and colleagues investigated the effects from cognitive dual task training on gait function in patients with PD. A decreased double support time was found following a 12 sessions program (30 min each session, three sessions per week for 4 weeks); furthermore, motor dual task gait training led to decreased gait variability in motor dual task walking conditions [101].

Finally, the application of noninvasive brain stimulation techniques, such as repetitive transcranial magnetic stimulation (rTMS) and transcranial direct current stimulation (tDCS), may maximize clinical benefits through mechanisms involving cortical plasticity $[104,105]$. The underlying molecular basis is not fully known, but it seems to involve synaptic remodeling through LTP and long-term depression (LTD) phenomena [106].

Manenti et al., [107] investigated the effects of anodal transcranial direct current stimulation (tDCS) over the dorsolateral prefrontal cortex in combination with physical therapy in PD patients. Patients were either assigned to anodal tDCS plus physical therapy (2 weeks of treatment consisting of daily direct current stimulation application for 25 min during physical therapy), or to sham neuromodulation plus physical therapy. While significant improvements in motor skills and depressive symptoms were observed in both groups, performances on PD Cognitive Rating Scale and verbal fluency tests improved only following add-on neuromodulation. These benefits were sustained 
over time (3 months). A number of studies emphasized the potential of tDCS and rTMS to modulate both motor and cognitive functions [108-111] and to improve neuroplasticity [104,105].

Interestingly, add-on tDCS in conjunction with dual task gait training may positively influence cognitive performances while walking under certain experimental conditions [112]. The effect of tDCS and rTMS on prefrontal cortex $[108,113,114]$ could be related to modulation of functional connectivity on corticostriatal level, thus somehow replicating what is observed with rehabilitative interventions based on motor-cognitive training. This provides further evidence for motor benefits that are mediated, at least partially, by neuroplastic phenomena.

Using different behavioral tasks probing reaction time (RTs), Ortelli et al., [115] investigated the relationship between asymmetric dopaminergic degeneration and attentional resources in PD patients undergoing motorcognitive rehabilitation. Attention-mediated performances did not significantly differ between right-side (RPD) and left-side (LPD) predominantly affected patients. However, only LPD patients showed significant improvements on attentional tasks following motor-cognitive rehabilitation. The authors hypothesized that these different profiles of cognitive modifiability in PD patients may be related to a lateralized susceptibility of the nigrostriatal system to neuronal degeneration and brain plasticity [115]. Importantly, these findings were in agreement with a previous randomized controlled trial describing the effects of 24 weeks of structured exercise interventions in PD [116]. The study provided Class IV level evidence for Progressive Resistance Exercise or Modified Fitness Counts in improving attention and working memory in non-demented PD patients with mild-to-moderate disease severity.

\section{Aerobic exercise in PD: scientific rationale \& clinical evidence}

Solid experimental evidence suggests that physical exercise may promote brain plasticity through the activation of neurotrophin signaling pathways [117], synaptogenesis [118], angiogenesis and neurogenesis [119,120]. Furthermore, beneficial effects from aerobic exercises may be mediated by reduced neuroinflammation [121] oxidative stress [122], and by improved intracellular calcium homeostasis [123].

In PD models, aerobic exercise may induce compensatory rearrangements of dopamine neurotransmissions by virtue of neuroplastic changes involving the striatal-thalamic-cortical motor circuits [32,124-127]. In neurotoxinbased models of PD, such as MPTP and 6-OHDA, physical exercise facilitates dopamine release (DA) through the modulation of DA receptors $[124,128,129]$. Using western immunoblotting analysis of synaptoneurosomes and in vivo positron emission tomography imaging with DA-D2R specific ligand $\left[{ }^{18} \mathrm{~F}\right]$ fallypride, Vučković et al., [130] observed an increase in striatal DA-D2R expression within dorsal striatum in MPTP mice following treadmill training. Notably, the increase expression of DA-D2R in the dorsal striatum has been linked to the potential restoration of automatic motor patterns in PD models [32].

It is hypothesized that exercise-induced effects on brain plasticity may also involve other non-dopaminergic circuitries. Consolidated evidence from in vitro and animal models shows that following dopamine depletion, a glutamate overdrive within corticostriatal connections tends to emerge. This phenomenon has been linked to aberrant learning and memory processes [131]. In neurotoxin-based rodent models of PD, exercise seems able to reverse this aberrant hyperactive glutamatergic state by reducing the presynaptic release of glutamate. Interestingly, these changes are accompanied by changes in the firing pattern of nigral dopamine neurons, suggesting a strong correlation between these two neurochemical systems in PD [123,132].

In addition to changes in neurotransmitters, modulation of neurotrophic factors may play an important role in the exercise-induced neuroplastic changes. These proteins are pivotal in key neurobiological processes including neuronal survival, growth and synaptogenesis $[133,134]$. Specifically, the action of BDNF is a candidate mechanism underlying exercise-induced benefits. Literature data suggest that BDNF release can help to optimize brain plasticity outcomes via exercise interventions, which could be properly relevant in the context of multimodal training (i.e., exercise and cognitive stimulation) [135]. Interestingly, in the context of exercise, the candidate tissues for the addition of BDNF to circulation is not only the brain, but also skeletal muscle, peripheral blood mononuclear cells, vascular endothelial cells and platelets via the spleen [135]. In particular, endothelial cells rapidly secrete BDNF in proportion to the magnitude of exercise-like stimuli, including shear stress [136] and reductions in $\mathrm{PO}_{2}$ [137]. Consistently with the upregulation of central BDNF expression in rodents, physical activity was found to increase circulating BDNF levels in both healthy humans and PD patients [138-140]. Fontanesi et al., [140] tested the hypothesis that a 4-week rehabilitation program, including aerobic exercise and functional and goal-directed training, could enhance BDNF-TrkB signaling in lymphocytes in patients with PD (Hoehn \& Yahr stage 23). Following the intervention, a significant improvement in motor and non-motor symptoms along with an up-regulation of BDNF-TrkB signaling [140] was observed. Moreover, changes in the Unified Parkinson's Disease 
Rating Scale significantly correlated with the increase in TrkB signaling, thus suggesting that clinical benefits in this population may be mediated by enhanced BDNF-TrkB signaling in lymphocyte. Two randomized controlled trials tested the rehabilitation effects on functional outcomes in PD and whether the treatment increases the BDNF serum levels [141]. Frazzitta et al., [142] enrolled 30 participants in early stages of PD who were assigned to intensive rehabilitation or to a control group (no rehabilitation). The intervention lasted 28 days and included aerobic exercise. The authors found that the intervention increased the BDNF levels and improved PD signs [142]. Sajatovic et al., [143] aimed to compare changes in depression in people with PD with comorbid depression between individual versus group exercise plus chronic disease self-management. The authors selected some biomarkers of inflammation and neuronal integrity, including BDNF, as outcome measures possibly related to mechanisms involved in depression. They found a significant increase in plasma BDNF level that corresponded to the initial 12-week 'intensive' portion of the interventions [143].

Similarly to what observed in animal models, Fisher and colleagues [144] reported an increased in DA-D2R binding potential within the dorsal striatum of individuals with early stage PD following an 8 weeks training program. Interestingly, these findings specifically correlated with improved postural control, suggesting that benefits from exercise may also be task-specific. Other studies seem to indicate dose-dependent effects from physical activity. However, the specific role played by motor tasks and dose in influencing the final clinical outcomes remains a matter of investigation. Finally, mounting evidence from different neurophysiologic studies suggest that high-intensity exercise may normalize corticomotor excitability in early PD [145]. These findings are consistent with the wellknown relationship between exercise intensity and BDNF levels, with higher exercise intensities inducing larger BDNF increases [146]: noteworthy, low to moderate intensity exercise is less effective than high-intensity exercise at increasing BDNF concentrations in healthy adults $[147,148]$.

Overall, these findings in humans seem to recapitulate prior evidence from both in vitro and animal models, suggesting that neuroplastic re-arrangements following intensive exercise may be mediated though different, potentially interconnected molecular mechanisms $[124,129]$.

For the scopes of the present perspective, it is noteworthy that exercise paradigms incorporating aerobic training in PD may improve cognition and motor learning in addition to their known effects on motor function $[32,149]$.

Indeed, the dopaminergic neuronal loss in the basal ganglia may directly affect cognitive functions with specific respect to executive domains [150,151]. Different studies with functional magnetic resonance imaging suggest that aerobic exercise may increase connectivity between brain regions specifically involved in affect, reward, learning, memory, attention and executive control [152]. In this setting, Duchesne et al., [153] described a functional reorganization of brain activity in cerebral regions concerned with motor learning (hippocampus, striatum and cerebellum) in 19 early stage PD patients following a 12-week progressive aerobic training. These functional changes were accompanied by improvements on behavioral outcomes observed in PD patients. Together with additional data showing improved executive functions following aerobic exercise [154], current evidence suggests that cognitive benefits following physical activity could be mediated by enhanced activation in frontal brain regions.

Silveira et al., [155] compared the effects of aerobic and goal-based exercise on five cognitive domains (attention and working memory, executive functions, memory, language and visuospatial function) in cognitively normal and impaired individuals with PD who were randomly allocated into three intervention groups: aerobic, goal-based and control. The authors found that aerobic exercise was more effective than goal-based exercise in improving executive functions (i.e., inhibitory control) in both cognitively normal and impaired individuals with PD.

These findings are extremely relevant in light of the role played by frontal brain regions in modulating attentive processes putatively used to compensate for impaired motor automaticity in PD [37].

\section{Integrating motor-cognitive rehabilitation with aerobic exercise: open questions}

Mounting evidence from clinical, behavioral, brain imaging, animal models and in vitro studies, supports the combined use of intensive, 'goal-based', motor-cognitive practices with aerobic training to achieve greater and more sustained clinical benefits in patients with PD (Figure 2) [32,37,134]. In the attempt to optimize complementary models of care for PD, we believe that future rehabilitative approaches should integrate both motor-cognitive and aerobic interventions. While cognitive engagement seems critical to achieve motor-behavioral benefits, aerobic training may act synergistically by maximizing brain plasticity. Indeed, this perspective of care encompasses all the main strategies historically adopted in PD rehabilitation: cueing techniques [156], use of feedback [157], verbal instructions [158] external focus [75], MI [159,160], biofeedback [161], AOT [96,160], mechanical devices [85,86], VR [92,92], non-invasive brain stimulation techniques [104,105], front-to-front physical therapy [25], aquatic therapy [162,163], 


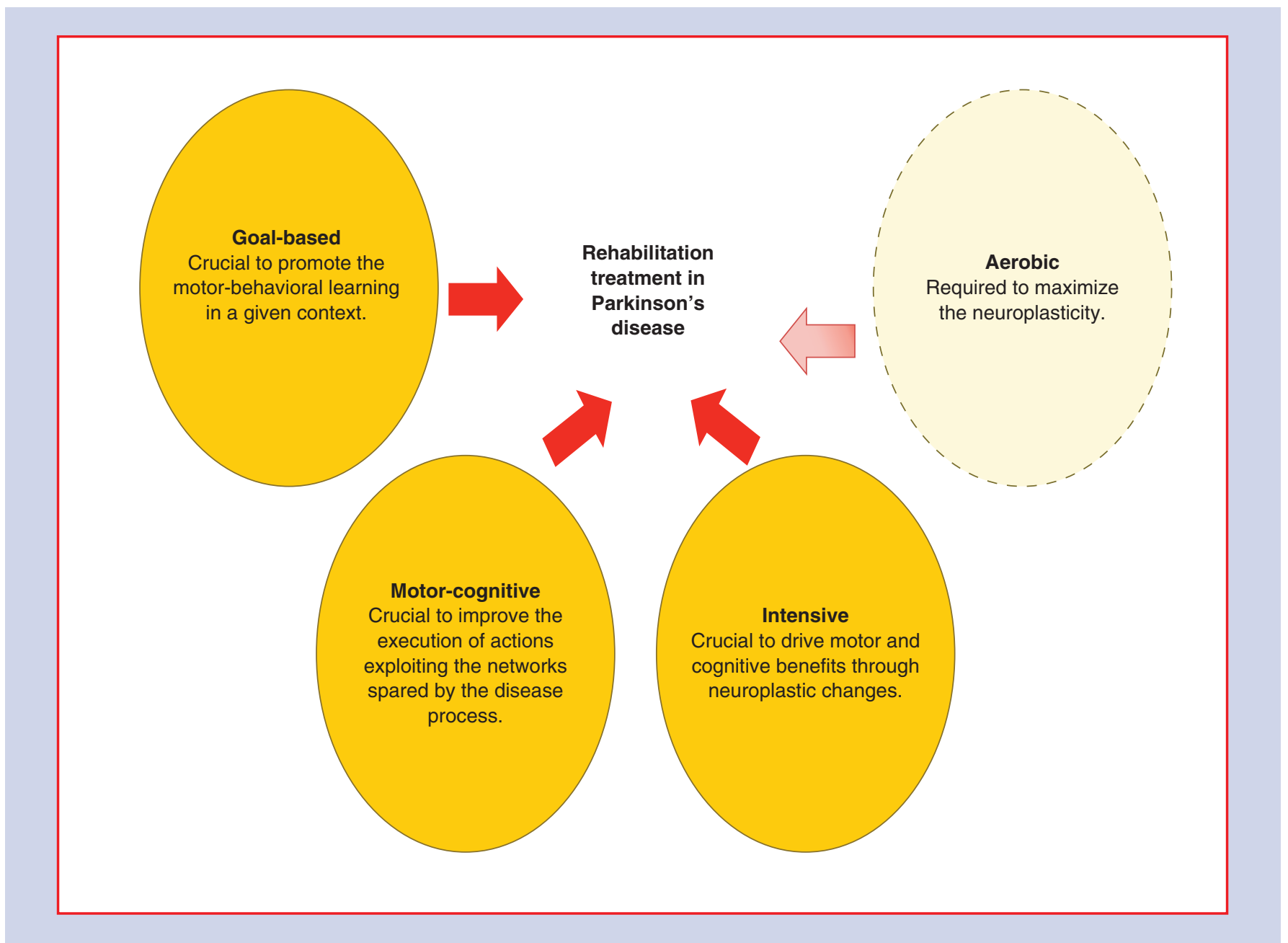

Figure 2. The theories on which rehabilitation in Parkinson disease should be based.

Combining an intensive and 'goal-based', motor-cognitive practice with aerobic training promotes neuroplasticity at the corticostriatal level, stimulates the executive resources and promotes the learning processes, thus probably representing the best way to obtain sustained improvements in Parkinson's disease (see both of the paragraphs about motor-cognitive intervention and aerobic exercise in Parkinson's disease).

speech and swallowing therapies [61] and occupational therapy [62]. Cognitive engagement may play an equally important role in other complementary activities designed for patients with PD, including dance therapy [164,165], art therapy and music therapy [166].

Future personalized protocols aiming to integrate these modalities of rehabilitation should also identify optimal parameters of intensity, frequency, duration and task-specific exercises to improve effectiveness and tolerability. This element introduces the question regarding the actual level of standardization and reproducibility of complementary interventions in PD. Although the current methodological heterogeneity of published studies prevents from pooling the available evidence to generate conclusive recommendations, growing evidence seems to confirm and converge on the use of multidisciplinary models of care [25,26,167-171] involving different health professionals including neurologists, physiatrists, physical therapists, occupational therapists, speech therapists, psychologists, nurses, social workers - among others. This approach may succeed in shifting the pendulum from the current construct of 'one disease - one disability' to more comprehensive and integrated strategies focusing on patient's individual complexity; and functional, familiar and social needs as a whole. Hopefully, different clinical, demographical and medical aspects (including comorbidities) should be weighted to adapt the intervention to the needing and personal motor and cognitive resources of each patient. For example, 'moving' the intervention toward the goal-based cognitive side, rather than to the aerobic side, could be useful for frail, advanced-stages patients. Conversely, the aerobic exercise, 
promoting plasticity, can be advantageously adopted in cognitively impaired patients for whom the cognitive, goal-based approach is hampered. Moreover, when the cognitive, goal-based approach is exploitable, it is crucial to evaluate which are the problems the patient presents in performing actions in a multi-dimension perspective (including the motor, motivational and cognitive aspects): how much the patient is motivated to perform the specific action (i.e., walking, or talking or writing)? Is it possible to increase his/her motivation? Does the patient know the correct strategies to manage the difficulties in action performing? Is it possible to modify his/her adopted strategies for ameliorating the motor performance? Which motor aspects are impaired? Finally, the time to spend in the different activities (occupational therapy, speech therapy, balance training, gait training, psychological therapy etc.) should be framed on the basis of what any single patient really needs. Therefore, algorithms to stratify the interventions and make them even more person-centered could be designed in the upcoming future. As a matter of fact, the multidisciplinary approach has been preliminarily reported to be effective in improving clinical conditions and QoL in PD [25,26,167-171], although further appropriately designed, well-powered studies are needed to corroborate these findings.

Results from these studies raise the question regarding the cost-benefit ratio and the practical applicability of these integrated interventions. Given the current demographic trend, the prevalence of PD is expected to exponentially increase in the next years, thus reaching a pandemic dimension [1]. The enormous social and economic impact for patients and their caregivers $[172,173]$ is aggravated by the aggregate burden of pharmacological therapies in terms of direct costs, adverse reactions and side effects [174,175]. Therefore, the cost saving effect of multifaceted comprehensive non-pharmacological approaches preserving long-term functionality and improving QoL may be outstanding, especially if compared with the aggregate cost deriving from addressing every patient's comorbidity separately. This favorable aspect is also partially suggested by prior studies reporting a reduced or controlled amount of dopaminergic medications administered to PD patients receiving dedicated rehabilitation programs [25,26]. These considerations should be included in economic models evaluating the cost-effectiveness of rehabilitation.

Further questions related to the optimal outcomes chosen to measure the aggregate effect of comprehensive and integrated neurorehabilitative approaches should also be addressed. Intuitively, the simple use of clinical tools biased toward appendicular motor function may not capture clinically significant improvements occurring in multiple, interdependent functional domains. Broader, comprehensive measures should be incorporated into both clinical practice and clinical trials, including kinematic, cognitive, psychological, emotional, behavioral and social outcomes. Moreover, only few studies evaluated cost-consequences analysis of rehabilitation in PD and many limitations stand [176]. Converging literature indicates that dedicated rehabilitative interventions are associated with fewer complications and lower costs in real world settings [177].

Finally, the optimal setting in which motor-cognitive rehabilitation and aerobic interventions may be administered remains to be defined. While an inpatient setting may be optimal to ensure treatments requiring higher intensity of care, home-based activities (possibly tele-monitored by electronic devices or smartphone applications) as well as outpatient programs, should be implemented to favor the maintenance of benefits over time. The coordination of different health professionals within dedicated networks and constant interactions with patients' communities through outreach programs may successfully serve to this purpose and should therefore be encouraged [178].

\section{Conclusion \& future perspective}

A large amount of data suggest that effective rehabilitative interventions for PD should incorporate motorcognitive training with aerobic activities and be designed accordingly. The specification of the chosen rehabilitative parameters, such as intensity, specificity and complexity, should always be formally disclosed in order to promote standardization and comparability of different protocols across dedicated centers. Future studies should address these methodological considerations in order to generate high quality experimental evidence (e.g., by comparing different protocols embracing the motor-cognitive interplay in randomized controlled trials) and lead to the identification of optimal models of care in PD. The development of comprehensive, patient-centered modalities of rehabilitation may succeed in harnessing long-lived clinical benefits and improvements in QoL in patients affected by this destructive disease. 


\section{Financial \& competing interests disclosure}

The authors have no relevant affiliations or financial involvement with any organization or entity with a financial interest in or financial conflict with the subject matter or materials discussed in the manuscript. This includes employment, consultancies, honoraria, stock ownership or options, expert testimony, grants or patents received or pending, or royalties.

No writing assistance was utilized in the production of this manuscript.

\section{Open access}

This work is licensed under the Attribution-NonCommercial-NoDerivatives 4.0 Unported License. To view a copy of this license, visit http://creativecommons.org/licenses/by-nc-nd/4.0/

\section{References}

Papers of special note have been highlighted as: • of interest $\bullet \bullet$ of considerable interest

1. Dorsey ER, Bloem BR. The Parkinson pandemic-a call to action. JAMA Neurol. 75(1), 9-10 (2018).

2. Lees AJ, Hardy J, Revesz T. Parkinson's disease. Lancet 373(9680), 2055-2066 (2009).

3. DeLong MR, Wichmann T. Circuits and circuit disorders of the basal ganglia. Arch. Neurol. 64(1), 20-24 (2007).

4. Prakash KM, Nadkarni NV, Lye WK, Yong MH, Tan EK. The impact of non-motor symptoms on the quality of life of Parkinson's disease patients: a longitudinal study. Eur. J. Neurol. 23(5), 854-860 (2016).

5. Groenewegen HJ. The basal ganglia and motor control. Neural. Plast. 10(1-2), 107-120 (2003).

6. Leisman G, Braun-Benjamin O, Melillo R. Cognitive-motor interactions of the basal ganglia in development. Front. Syst. Neurosci. 8, 16 (2014).

7. Yelnik J. Modeling the organization of the basal ganglia. Rev. Neurol. (Paris) 164(12), 969-976 (2008).

8. Lang AE, Obeso JA. Challenges in Parkinson's disease: restoration of the nigrostriatal dopamine system is not enough. Lancet Neurol. 3(5), 309-316 (2004).

9. Devos D, Defebvre L, Bordet R. Dopaminergic and non-dopaminergic pharmacological hypotheses for gait disorders in Parkinson's disease. Fundam. Clin. Pharmacol. 24(4), 407-421 (2010).

10. Schrag A, Jahanshahi M, Quinn N. What contributes to quality of life in patients with Parkinson's disease? J. Neurol. Neurosurg. Psychiatry 69(3), 308-312 (2000).

11. Cano-de-la-Cuerda R, Vela-Desojo L, Miangolarra-Page JC, Macías-Macías Y, Muñoz-Hellín E. Axial rigidity and quality of life in patients with Parkinson's disease: a preliminary study. Qual. Life Res. 20(6), 817-823 (2000).

12. Appleman ER, Stavitsky K, Cronin-Golomb A. Relation of subjective quality of life to motor symptom profile in Parkinson's disease. Parkinsons Dis. 2011, 5 (2011).

13. Martinez-Martin P, Rodriguez-Blazquez C, Forjaz MJ, Kurtis MM. Impact of pharmacotherapy on quality of life in patients with Parkinson's disease. CNS Drugs 29(5), 397-413 (2015).

14. Gallagher DA, Schrag A. Impact of newer pharmacological treatments on quality of life in patients with Parkinson's disease. CNS Drugs 22(7), 563-586 (2008).

15. Chapuis S, Ouchchane L, Metz O, Gerbaud L, Durif F. Impact of the motor complications of Parkinson's disease on the quality of life. Mov. Disord. 20(2), 224-230 (2005).

16. Cutson TM, Laub KC, Schenkman M. Pharmacological and nonpharmacological interventions in the treatment of Parkinson's disease. Phys. Ther. 75(5), 363-373 (1995).

17. Abbruzzese G, Marchese R, Avanzino L, Pelosin E. Rehabilitation for Parkinson's disease: Current outlook and future challenges. Parkinsonism Relat. Disord. 22(Suppl.1), S60-S64 (2016).

18. Comella CL, Stebbins GT, Brown-Toms N, Goetz CG. Physical therapy and Parkinson's disease: a controlled clinical trial. Neurology 44, 376-378 (1994).

19. Formisano R, Pratesi L, Modarelli FT, Bonifati V, Meco G. Rehabilitation and Parkinson's disease. Scand. J. Rehabil. Med. 24(3), 157-160 (1992).

20. Tomlinson CL, Patel S, Meek C et al. Physiotherapy versus placebo or no intervention in Parkinson's disease. Cochrane Database Syst. Rev. 9, CD002817 (2013).

21. Tomlinson CL, Herd CP, Clarke CE et al. Physiotherapy for Parkinson's disease: a comparison of techniques. Cochrane Database Syst. Rev. 6, CD002815 (2014)

22. Bloem BR, de Vries NM, Ebersbach G. Nonpharmacological treatments for patients with Parkinson's disease. Mov. Disord. 30(11), 1504-1520 (2015).

23. Goodwin VA, Richards SH, Taylor RS, Taylor AH, Campbell JL. The effectiveness of exercise interventions for people with Parkinson's disease: a systematic review and meta-analysis. Mov. Disord. 23(5), 631-640 (2008). 
24. Keus SH, Munneke M, Nijkrake MJ, Kwakkel G, Bloem BR. Physical therapy in Parkinson's disease: evolution and future challenges. Mov. Disord. 24(1), 1-14 (2009).

25. Frazzitta G, Maestri R, Bertotti G et al. Intensive rehabilitation treatment in early Parkinson's disease: a randomized pilot study with a 2-year follow-up. Neurorehabil. Neural Repair 29(2), 123-131 (2015).

26. Ferrazzoli D, Ortelli P, Zivi I et al. Efficacy of intensive multidisciplinary rehabilitation in Parkinson's disease: a randomised controlled study. J. Neurol. Neurosurg. Psychiatry 89(8), 828-835 (2018).

- Confirms the clinical benefits in patients with Parkinson's disease (PD) after a program of inpatient multidisciplinary rehabilitation and shows the related improvement in quality of life in the short and long-term.

27. Nagano-Saito A, Martinu K, Monchi O. Function of basal ganglia in bridging cognitive and motor modules to perform an action. Front. Neurosci. 8, 187 (2014).

28. Leisman G, Melillo R. The basal ganglia: motor and cognitive relationships in a clinical neurobehavioral context. Rev. Neurosci. 24(1), 9-25 (2013).

29. Middleton FA, Strick PL. Basal ganglia output and cognition: evidence from anatomical, behavioral, and clinical studies. Brain Cogn 42(2), 183-200 (2000).

30. Mazzoni P, Shabbott B, Cortés JC. Motor control abnormalities in Parkinson's disease. Cold Spring Harb. Perspect. Med. 2(6), a009282 (2012)

31. Nelson AB, Kreitzer AC. Reassessing models of basal ganglia function and dysfunction. Annu. Rev. Neurosci. 37, 117-1135 (2014).

32. Petzinger GM, Fisher BE, McEwen S, Beeler JA, Walsh JP, Jakowec MW. Exercise-enhanced neuroplasticity targeting motor and cognitive circuitry in Parkinson's disease. Lancet Neurol. 12(7), 716-726 (2013).

-. Explains the potential of goal-based training and aerobic activity to improve both cognitive and automatic components of motor control in PD through experience-dependent neuroplasticity.

33. Schenkman M, Cutson TM, Kuchibhatla M et al. Exercise to improve spinal flexibility and function for people with Parkinson's disease: a randomized, controlled trial. J. Am. Geriatr. Soc. 46(10), 1207-1216 (1998).

34. Ellis T, de Goede CJ, Feldman RG, Wolters EC, Kwakkel G, Wagenaar RC. Efficacy of a physical therapy program in patients with Parkinson's disease: a randomized controlled trial. Arch. Phys. Med. Rehabil. 86(4), 626-632 (2005).

35. Canning CG, Alison JA, Allen NE, Groeller H. Parkinson's disease: an investigation of exercise capacity, respiratory function, and gait. Arch. Phys. Med. Rehabil. 78(2), 199-207 (1997).

36. Dibble LE, Hale TF, Marcus RL, Droge J, Gerber JP, LaStayo PC. High-intensity resistance training amplifies muscle hypertrophy and functional gains in persons with Parkinson's disease. Mov. Disord. 21(9), 1444-1452 (2006).

37. Ferrazzoli D, Ortelli P, Madeo G, Giladi N, Petzinger GM, Frazzitta G. Basal ganglia and beyond: The interplay between motor and cognitive aspects in Parkinson's disease rehabilitation. Neurosci. Biobehav. Rev. 90, 294-308 (2018).

38. Weintraub D, Burn DJ. Parkinson's disease: the quintessential neuropsychiatric disorder. Mov. Disord. 26(6), 1022-1031 (2011).

39. Ahn DH, Lee YJ, Jeong JH, Kim YR, Park JB. The effect of post-stroke depression on rehabilitation outcome and the impact of caregiver type as a factor of post-stroke depression. Ann. Rehabil. Med. 39(1), 74-80 (2015).

40. Shahab S, Nicolici DF, Tang A, Katz P, Mah L. Depression predicts functional outcome in geriatric inpatient rehabilitation. Arch. Phys. Med. Rehabil. 98(3), 500-507 (2017).

41. Marsden CD. The mysterious motor function of the basal ganglia: the Robert Wartenberg Lecture. Neurology 32(5), 514-539 (1982).

42. Hoshiyama M, Kaneoke Y, Koike Y, Takahashi A, Watanabe S. Hypokinesia of associated movement in Parkinson's disease: a symptom in early stages of the disease. J. Neurol. 241(9), 517-521 (1994).

43. Wu T, Hallett M, Chan P. Motor automaticity in Parkinson's disease. Neurobiol. Dis. 82, 226-234 (2015).

44. Gilat M, Bell PT, Ehgoetz Martens KA et al. Dopamine depletion impairs gait automaticity by altering cortico-striatal and cerebellar processing in Parkinson's disease. Neuroimage 152, 207-220 (2017).

45. Frank MJ, Seeberger LC, O'reilly RC. By carrot or by stick: cognitive reinforcement learning in parkinsonism. Science 306(5703), 1940-1943 (2004).

46. Schott BH, Niehaus L, Wittmann BC et al. Ageing and early-stage Parkinson's disease affect separable neural mechanisms of mesolimbic reward processing. Brain 130, 2412-2424 (2007).

47. Shohamy D, Myers CE, Grossman S, Sage J, Gluck MA. The role of dopamine in cognitive sequence learning: evidence from Parkinson's disease. Behav. Brain Res. 156(2), 191-199 (2005).

48. Penhune VB, Steele CJ. Parallel contributions of cerebellar, striatal and M1 mechanisms to motor sequence learning. Behav. Brain Res. 226(2), 579-591 (2012).

49. Shiner T, Seymour B, Wunderlich K et al. Dopamine and performance in a reinforcement learning task: evidence from Parkinson's disease. Brain 135, 1871-1883 (2012).

50. Cools AR, van den Bercken JH, Horstink MW, van Spaendonck KP, Berger HJ. Cognitive and motor shifting aptitude disorder in Parkinson's disease. J. Neurol. Neurosurg. Psychiatry 47(5), 443-453 (1984). 
51. Cools R, Barker RA, Sahakian BJ, Robbins TW. Enhanced or impaired cognitive function in Parkinson's disease as a function of dopaminergic medication and task demands. Cereb. Cortex 11(12), 1136-1143 (2001).

52. Hikosaka O, Isoda M. Switching from automatic to controlled behavior: cortico-basal ganglia mechanisms. Trends. Cogn. Sci. 14(4), 154-161 (2010).

53. Kim HF, Hikosaka O. Parallel basal ganglia circuits for voluntary and automatic behaviour to reach rewards. Brain 138, 1776-1800 (2015).

- This is a relevant paper for understanding the functions and mechanisms of the basal ganglia parallel circuits and may inform the differential diagnosis and treatment of basal ganglia disorders.

54. Berardelli A, Rothwell JC, Thompson PD, Hallett M. Pathophysiology of bradykinesia in Parkinson's disease. Brain 124, 2131-2146 (2001).

55. Pieruccini-Faria F, Jones JA, Almeida QJ. Motor planning in Parkinson's disease patients experiencing freezing of gait: the influence of cognitive load when approaching obstacles. Brain Cogn. 87, 76-85 (2014).

56. Xu D, Cole MH, Mengersen K et al. Executive function and postural instability in people with Parkinson's disease. Parkinsons Dis. 2014, 684758 (2014).

57. Lindholm B, Hagell P, Hansson O, Nilsson MH. Prediction of falls and/or near falls in people with mild Parkinson's disease. PLoS ONE 10(1), e0117018 (2015).

58. Herman T, Mirelman A, Giladi N, Schweiger A, Hausdorff JM. Executive control deficits as a prodrome to falls in healthy older adults: a prospective study linking thinking, walking, and falling. J. Gerontol. A. Biol. Sci. Med. Sci. 65, 1086-1092 (2010).

59. Wagle Shukla A, Ounpraseuth S, Okun MS, Gray V, Schwankhaus J, Metzer WS. Micrographia and related deficits in Parkinson's disease: a cross-sectional study. BMJ Open 2(3), e000628 (2012).

60. Dashtipour K, Tafreshi A, Lee J, Crawley B. Speech disorders in Parkinson's disease: pathophysiology, medical management and surgical approaches. Neurodegener. Dis. Manag. 8(5), 337-348 (2018).

61. Tjaden K. Speech and swallowing in Parkinson's Disease. Top. Geriatr. Rehabil. 24(2), 115-126 (2008).

62. Radder DLM, Sturkenboom IH, van Nimwegen M, Keus SH, Bloem BR, de Vries NM. Physical therapy and occupational therapy in Parkinson's disease. Int. J. Neurosci. 127(10), 930-943 (2017).

63. Kearney E, Haworth B, Scholl J, Faloutsos P, Baljko M, Yunusova Y. Treating speech movement hypokinesia in Parkinson's disease: does movement size matter? J. Speech Lang. Hear. Res. 61(11), 2703-2721 (2018).

64. Smith JG, McDowall J. The implicit sequence learning deficit in patients with Parkinson's disease: a matter of impaired sequence integration? Neuropsychologia 44(2), 275-288 (2006).

65. Siegert RJ, Taylor KD, Weatherall M, Abernethy DA. Is implicit sequence learning impaired in Parkinson's disease? A meta-analysis. Neuropsychology 20(4), 490-495 (2006).

66. Nieuwboer A, Rochester L, Müncks L, Swinnen SP. Motor learning in Parkinson's disease: limitations and potential for rehabilitation. Parkinsonism Relat. Disord. 15(Suppl. 3), S53-S58 (2009).

-. Supports the notion that adopting motor learning principles could benefit rehabilitation in PD.

67. Dirnberger G, Jahanshahi M. Executive dysfunction in Parkinson's disease: a review. J. Neuropsychol. 7(2), 193-224 (2013).

68. Kudlicka A, Clare L, Hindle JV. Executive functions in Parkinson's disease: systematic review and meta-analysis. Mov. Disord. 26(13), 2305-2315 (2011).

69. Lehman DA, Toole T, Lofald D, Hirsch MA. Training with verbal instructional cues results in near-term improvement of gait in people with Parkinson disease. J. Neurol. Phys. Ther. 29(1), 2-8 (2005).

70. Morris ME, Iansek R, Galna B. Gait festination and freezing in Parkinson's disease: pathogenesis and rehabilitation. Mov. Disord. 23(Suppl. 2), S451-S460 (2008).

71. Morris ME, Martin CL, Schenkman ML. Striding out with Parkinson disease: evidence-based physical therapy for gait disorders. Phys. Ther. 90(2), 280-288 (2010).

72. Morris ME. Locomotor training in people with Parkinson disease. Phys. Ther. 86(10), 1426-1435 (2006).

73. Ferrazzoli D, Ortelli P, Maestri R et al. Focused and sustained attention is modified by a goal-based rehabilitation in Parkinsonian patients. Front. Behav. Neurosci. 11, 56 (2017).

74. Lohnes CA, Earhart GM. The impact of attentional, auditory, and combined cues on walking during single and cognitive dual tasks in Parkinson disease. Gait Posture 33(3), 478-483 (2011).

75. Wulf G, Landers M, Lewthwaite R, Töllner T. External focus instructions reduce postural instability in individuals with Parkinson disease. Phys. Ther. 89(2), 162-168 (2009).

76. Shima K, Isoda M, Mushiake H, Tanji J. Categorization of behavioural sequences in the prefrontal cortex. Nature 445, 315-318 (2007).

77. Svoboda K, Li N. Neural mechanisms of movement planning: motor cortex and beyond. Curr. Opin. Neurobiol. 49, 33-41 (2018).

78. Sakagami M, Pan X. Functional role of the ventrolateral prefrontal cortex in decision making. Curr. Opin. Neurobiol. 17(2), 228-233 (2007). 
79. Redgrave P, Rodriguez M, Smith Y et al. Goal-directed and habitual control in the basal ganglia: implications for Parkinson's disease. Nat. Rev. Neurosci. 11(11), 760-772 (2010).

-. Explains how many of the behavioral difficulties in PD reflect the loss of normal automatic control and the need to rely on goal-directed mode of action.

80. Nombela C, Hughes LE, Owen AM, Grahn JA. Into the groove: can rhythm influence Parkinson's disease? Neurosci. Biobehav. Rev. 37 , 2564-2570 (2013).

81. Boettiger CA, D’Esposito M. Frontal networks for learning and executing arbitrary stimulus-response associations. J. Neurosci. 25(10), 2723-2732 (2005).

82. Kearsley G. Explorations in Learning \& Instruction: The Theory into Practice Database (2006). http://158.132.155.107/posh97/ private/TIP/3.htm

83. Seger CA. How do the basal ganglia contribute to categorization? Their roles in generalization, response selection, and learning via feedback. Neurosci. Biobehav. Rev. 32(2), 265-278 (2008).

84. McNevin NH, Wulf G, Carlson C. Effects of attentional focus, self-control, and dyad training on motor learning: implications for physical rehabilitation. Phys. Ther. 80(4), 373-385 (2000).

85. Frenkel-Toledo S, Giladi N, Peretz C, Herman T, Gruendlinger L, Hausdorff JM. Treadmill walking as an external pacemaker to improve gait rhythm and stability in Parkinson's disease. Mov. Disord. 20(9), 1109-1114 (2005).

- Suggests that the treadmill may be acting as an external cue to enhance gait rhythmicity and reduce gait variability in PD.

86. Frazzitta G, Maestri R, Uccellini D, Bertotti G, Abelli P. Rehabilitation treatment of gait in patients with Parkinson's disease with freezing: a comparison between two physical therapy protocols using visual and auditory cues with or without treadmill training. Mov. Disord. 24(8), 1139-1143 (2009).

-• Suggests that treadmill training associated with auditory and visual cues might give better results than more conventional treatments.

87. Calabrò RS, Naro A, Filoni S et al. Walking to your right music: a randomized controlled trial on the novel use of treadmill plus music in Parkinson's disease. J. Neuroeng. Rehabil. 16(1), 68 (2019).

88. Sveistrup H. Motor rehabilitation using virtual reality. J. Neuroeng. Rehabil. 1(1), 10 (2004).

89. Bisson E, Contant B, Sveistrup H, Lajoie Y. Functional balance and dual-task reaction times in older adults are improved by virtual reality and biofeedback training. Cyberpsychol. Behav. 10(1), 16-23 (2007).

90. Mirelman A, Maidan I, Deutsch JE. Virtual reality and motor imagery: promising tools for assessment and therapy in Parkinson's disease. Mov. Disord. 28(11), 1597-1608 (2013).

91. Mirelman A, Maidan I, Herman T, Deutsch JE, Giladi N, Hausdorff JM. Virtual reality for gait training: can it induce motor learning to enhance complex walking and reduce fall risk in patients with Parkinson's disease? J. Gerontol. A. Biol. Sci. Med. Sci. 66(2), 234-240 (2011).

92. Mirelman A, Rochester L, Maidan I et al. Addition of a non-immersive virtual reality component to treadmill training to reduce fall risk in older adults (V-TIME): a randomised controlled trial. Lancet 388(10050), 1170-1182 (2016).

- Shows that treadmill training plus virtual reality led to reduced fall rates compared with treadmill training alone in a diverse group of older adults at high risk for falls.

93. Cunnington R, Egan GF, O’Sullivan JD, Hughes AJ, Bradshaw JL, Colebatch JG. Motor imagery in Parkinson's disease: a PET study. Mov. Disord. 16(5), 849-857 (2001).

94. Caligiore D, Mustile M, Spalletta G, Baldassarre G. Action observation and motor imagery for rehabilitation in Parkinson's disease: A systematic review and an integrative hypothesis. Neurosci. Biobehav. Rev. 72, 210-222 (2017).

95. Rizzolatti G, Fogassi L, Gallese V. Neurophysiological mechanisms underlying the understanding and imitation of action. Nat. Rev. Neurosci. 2(9), 661-670 (2011).

96. Pelosin E, Barella R, Bet C et al. Effect of group-based rehabilitation combining action observation with physiotherapy on freezing of gait in Parkinson's disease. Neural. Plast. 2018, 4897276 (2018).

97. Agosta F, Gatti R, Sarasso E et al. Brain plasticity in Parkinson's disease with freezing of gait induced by action observation training. J. Neurol. 264(1), 88-101 (2017).

98. Strouwen C, Molenaar EA, Münks L et al. Dual tasking in Parkinson's disease: should we train hazardous behavior? Expert Rev. Neurother. 15, 1031-1039 (2015).

99. Yogev G, Giladi N, Peretz C, Springer S, Simon ES, Hausdorff JM. Dual tasking, gait rhythmicity, and Parkinson's disease: which aspects of gait are attention demanding? Eur. J. Neurosci. 22(5), 1248-1256 (2005).

100. Heinzel S, Maechtel M, Hasmann SE et al. Motor dual-tasking deficits predict falls in Parkinson's disease: a prospective study. Parkinsonism Relat. Disord. 26, 73-77 (2016).

101. Yang YR, Cheng SJ, Lee YJ, Liu YC, Wang RY. Cognitive and motor dual task gait training exerted specific training effects on dual task gait performance in individuals with Parkinson's disease: a randomized controlled pilot study. PLoS ONE 14(6), e0218180 (2019). 
102. Fritz NE, Cheek FM, Nichols-Larsen DS. Motor-cognitive dual-task training in persons with neurologic disorders: a systematic review. J. Neurol. Phys. Ther. 39(3), 142-153 (2015).

103. Liu YC, Yang YR, Tsai YA, Wang RY. Cognitive and motor dual task gait training improve dual task gait performance after stroke - A randomized controlled pilot trial. Sci. Rep. 7(1), 4070 (2017).

104. Benninger DH, Lomarev M, Lopez G et al. Transcranial direct current stimulation for the treatment of Parkinson's disease. J. Neurol. Neurosurg. Psychiatry 81(10), 1105-1111 (2010).

105. Kamble N, Netravathi M, Pal PK. Therapeutic applications of repetitive transcranial magnetic stimulation (rTMS) in movement disorders: a review. Parkinsonism Relat. Disord. 20(7), 695-707 (2014).

106. Cucca A, Biagioni MC, Fleisher JE et al. Freezing of gait in Parkinson's disease: from pathophysiology to emerging therapies. Neurodegener. Dis. Manag. 6(5), 431-446 (2016).

107. Manenti R, Brambilla M, Benussi A et al. Mild cognitive impairment in Parkinson's disease is improved by transcranial direct current stimulation combined with physical therapy. Mov. Disord. 31(5), 715-724 (2016)

108. Manenti R, Brambilla M, Rosini $S$ et al. Time up and go task performance improves after transcranial direct current stimulation in patient affected by Parkinson's disease. Neurosci. Lett. 580, 74-77 (2014).

109. Boggio PS, Ferrucci R, Rigonatti SP et al. Effects of transcranial direct current stimulation on working memory in patients with Parkinson's disease. J. Neurol. Sci. 249(1), 31-38 (2006).

110. Ishikuro K, Dougu N, Nukui T et al. Effects of transcranial direct current stimulation (tDCS) over the frontal polar area on motor and executive functions in Parkinson's disease: a pilot study. Front. Aging Neurosci. 10, 231 (2018).

111. Goodwill AM, Lum JAG, Hendy AM et al. Using non-invasive transcranial stimulation to improve motor and cognitive function in Parkinson's disease: a systematic review and meta-analysis. Sci. Rep. 7(1), 14840 (2017).

112. Schabrun SM, Lamont RM, Brauer SG. Transcranial Direct Current Stimulation to Enhance Dual-Task Gait Training in Parkinson's Disease: A Pilot RCT. PLoS ONE 11, e0158497 (2016).

113. Dagan M, Herman T, Mirelman A, Giladi N, Hausdorff JM. The role of the prefrontal cortex in freezing of gait in Parkinson's disease: insights from a deep repetitive transcranial magnetic stimulation exploratory study. Exp. Brain Res. 235(8), 2463-2472 (2017).

114. Randver R. Repetitive transcranial magnetic stimulation of the dorsolateral prefrontal cortex to alleviate depression and cognitive impairment associated with Parkinson's disease: A review and clinical implications. J. Neurol. Sci. 393, 88-99 (2018).

115. Ortelli P, Ferrazzoli D, Zarucchi M, Maestri R, Frazzitta G. Asymmetric dopaminergic degeneration and attentional resources in Parkinson's disease. Front. Neurosci. 12, 972 (2018).

116. David FJ, Robichaud JA, Leurgans SE et al. Exercise improves cognition in Parkinson's disease: The PRET-PD randomized, clinical trial. Mov. Disord. 30(12), 1657-1663 (2015).

-. Demonstrates that exercise may improve attention and working memory in nondemented patients with mild-to-moderate PD.

117. Phillips C, Baktir MA, Srivatsan M, Salehi A. Neuroprotective effects of physical activity on the brain: a closer look at trophic factor signaling. Front. Cell. Neurosci. 8, 170 (2014).

118. Ambrogini P, Lattanzi D, Ciuffoli S, Betti M, Fanelli M, Cuppini R. Physical exercise and environment exploration affect synaptogenesis in adult-generated neurons in the rat dentate gyrus: possible role of BDNF. Brain Res. 1534, 1-12 (2013).

119. van Praag H, Shubert T, Zhao C, Gage FH. Exercise enhances learning and hippocampal neurogenesis in aged mice. J. Neurosci. 25(38), 8680-8685 (2005).

120. van der Borght K, Kóbor-Nyakas DE, Klauke K et al. Physical exercise leads to rapid adaptations in hippocampal vasculature: temporal dynamics and relationship to cell proliferation and neurogenesis. Hippocampus 19(10), 928-936 (2009).

121. Real CC, Garcia PC, Britto LRG. Treadmill exercise prevents increase of neuroinflammation markers involved in the dopaminergic damage of the 6-OHDA Parkinson's disease model. J. Mol. Neurosci. 63(1), 36-49 (2017).

122. Hemmati-Dinarvand M, Saedi S, Valilo M et al. Oxidative stress and Parkinson's disease: conflict of oxidant-antioxidant systems. Neurosci. Lett. 709, 134296 (2019).

123. Chen W, Qiao D, Liu X, Shi K. Treadmill exercise improves motor dysfunction and hyperactivity of the corticostriatal glutamatergic pathway in rats with 6-OHDA-induced Parkinson's disease. Neural. Plast. 2017, 2583910 (2017).

124. Petzinger GM, Walsh JP, Akopian G et al. Effects of treadmill exercise on dopaminergic transmission in the 1-methyl-4-phenyl-1,2,3,6-tetrahydropyridine-lesioned mouse model of basal ganglia injury. J. Neurosci. 27(20), 5291-5300 (2007).

125. Petzinger GM, Fisher BE, Van Leeuwen JE et al. Enhancing neuroplasticity in the basal ganglia: the role of exercise in Parkinson's disease. Mov. Disord. 25(Suppl 1.), S141-S145 (2010).

-. Presents findings from patients with PD and animal models and indicates that activity-dependent (exercise) processes, may mitigate the cortically driven hyper-excitability in the basal ganglia normally observed in the parkinsonian state.

126. Petzinger GM, Holschneider DP, Fisher BE et al. The effects of exercise on dopamine neurotransmission in Parkinson's disease: targeting neuroplasticity to modulate basal ganglia circuitry. Brain Plast. 1(1), 29-39 (2015).

127. Tajiri N, Yasuhara T, Shingo T et al. Exercise exerts neuroprotective effects on Parkinson's disease model of rats. Brain Res. 1310, 200-207 (2015). 
128. Petzinger GM, Fisher BE, Akopian G et al. The role of exercise in facilitating basal ganglia function in Parkinson's disease. Neurodegener. Dis. Manag. 1(2), 157-170 (2011).

129. Fisher BE, Petzinger GM, Nixon K et al. Exercise-induced behavioral recovery and neuroplasticity in the1-methyl-4-phenyl-1,2,3,6 tetrahydropyridine-lesioned mouse basal ganglia. J. Neurosci. Res. 77, 378-390 (2004).

130. Vučković MG, Li Q, Fisher B et al. Exercise elevates dopamine D2 receptor in a mouse model of Parkinson's disease: in vivo imaging with $\left[{ }^{18}\right.$ F]fallypride. Mov. Disord. 25(16), 2777-2784 (2010).

131. Calabresi P, Mercuri NB, Sancesario G, Bernardi G. Electrophysiology of dopamine-denervated striatal neurons. Implications for Parkinson's disease. Brain 116, 433-452 (1993).

132. Kintz N, Petzinger GM, Akopian G et al. Exercise modifies $\alpha$-amino-3-hydroxy-5-methyl-4-isoxazolepropionic acid receptor expression in striatopallidal neurons in the 1-methyl-4-phenyl-1,2,3,6-tetrahydropyridine-lesioned mouse. J. Neurosci. Res. 91(11), 1492-1507 (2013).

133. da Silva PG, Domingues DD, de Carvalho LA, Allodi S, Correa CL. Neurotrophic factors in Parkinson's disease are regulated by exercise: Evidence-based practice. J. Neurol. Sci. 363, 5-15 (2016).

134. Hirsch MA, Iyer SS, Sanjak M. Exercise-induced neuroplasticity in human Parkinson's disease: what is the evidence telling us? Parkinsonism Relat. Disord. 22(Suppl. 1), S78-S81 (2016).

135. Walsh JJ, Tschakovsky ME. Exercise and circulating BDNF: Mechanisms of release and implications for the design of exercise interventions. Appl. Physiol. Nutr. Metab. 43(11), 1095-1104 (2018).

136. Prigent-Tessier A, Quirié A, Maguin-Gaté K et al. Physical training and hypertension have opposite effects on endothelial brain-derived neurotrophic factor expression. Cardiovasc. Res. 100(3), 374-382 (2013).

137. Helan M, Aravamudan B, Hartman WR et al. BDNF secretion by human pulmonary artery endothelial cells in response to hypoxia. $J$. Mol. Cell. Cardiol. 68, 89-97 (2014).

138. Zoladz JA, Pilc A. The effect of physical activity on the brain derived neurotrophic factor: from animal to human studies. J. Physiol. Pharmacol. 61(5), 533-541 (2010).

139. Frazzitta G, Maestri R, Ghilardi MF et al. Intensive rehabilitation increases BDNF serum levels in parkinsonian patients: a randomized study. Neurorehabil. Neural Repair 28(2), 163-168 (2014).

140. Fontanesi C, Kvint S, Frazzitta G et al. Intensive Rehabilitation Enhances Lymphocyte BDNF-TrkB Signaling in Patients With Parkinson's Disease. Neurorehabil. Neural Repair 30(5), 411-418 (2016).

141. Hirsch MA, van Wegen EEH, Newman MA, Heyn PC. Exercise-induced increase in brain-derived neurotrophic factor in human Parkinson's disease: a systematic review and meta-analysis. Transl. Neurodegener. 7, 7 (2018).

142. Frazzitta G, Maestri R, Ghilardi MF et al. Intensive rehabilitation increases BDNF serum levels in parkinsonian patients: a randomized study. Neurorehabil. Neural Repair 28(2), 163-168 (2014).

143. Sajatovic M, Ridgel AL, Walter EM et al. A randomized trial of individual versus group-format exercise and self-management in individuals with Parkinson's disease and comorbid depression. Patient Prefer. Adherence 11, 965-973 (2017).

144. Fisher BE, Li Q, Nacca A et al. Treadmill exercise elevates striatal dopamine D2 receptor binding potential in patients with early Parkinson's disease. Neuroreport 24(10), 509-514 (2013).

145. Fisher BE, Wu AD, Salem GJ et al. The effect of exercise training in improving motor performance and corticomotor excitability in people with early Parkinson's disease. Arch. Phys. Med. Rehabil. 89(7), 1221-1229 (2008).

- Suggests the dose-dependent benefits of exercise and that high-intensity exercise can normalize corticomotor excitability in early PD.

146. Piepmeier AT, Etnier JL. Brain-derived neurotrophic factor (BDNF) as a potential mechanism of the effects of acute exercise on cognitive performance. J Sport Health Sci. 4(1), 14-23 (2015).

147. Knaepen K, Goekint M, Heyman EM, Meeusen R. Neuroplasticity - exercise-induced response of peripheral brain-derived neurotrophic factor: a systematic review of experimental studies in human subjects. Sports Med. 40(9), 765-801 (2010).

148. Jiménez-Maldonado A, Rentería I, García-Suárez PC, Moncada-Jiménez J, Freire-Royes LF. The impact of high-intensity interval training on brain derived neurotrophic factor in brain: a mini-review. Front. Neurosci. 12, 839 (2018).

149. Intzandt B, Beck EN, Silveira CRA. The effects of exercise on cognition and gait in Parkinson's disease: A scoping review. Neurosci. Biobehav. Rev. 95, 136-169 (2018).

150. Ekman U, Eriksson J, Forsgren L, Mo SJ, Riklund K, Nyberg L. Functional brain activity and presynaptic dopamine uptake in patients with Parkinson's disease and mild cognitive impairment: a cross-sectional study. Lancet Neurol. 11(8), 679-687 (2012).

151. Lewis SJ, Dove A, Robbins TW, Barker RA, Owen AM. Cognitive impairments in early Parkinson's disease are accompanied by reductions in activity in frontostriatal neural circuitry. J. Neurosci. 23(15), 6351-6356 (2003).

152. Weng TB, Pierce GL, Darling WG, Falk D, Magnotta VA, Voss MW. The acute effects of aerobic exercise onthe functional connectivity of human brain networks. Brain Plast. 2(2), 171-190 (2017).

153. Duchesne C, Gheysen F, Bore A et al. Influence of aerobic exercise training on the neural correlates of motor learning in Parkinson's disease individuals. Neuroimage Clin. 12, 559-569 (2016). 
154. Tabak R, Aquije G, Fisher BE. Aerobic exercise to improve executive function in Parkinson disease: a case series. J. Neurol. Phys. Ther. 37(2), 58-64 (2013).

155. Silveira CRA, Roy EA, Intzandt BN, Almeida QJ. Aerobic exercise is more effective than goal-based exercise for the treatment of cognition in Parkinson's disease. Brain Cogn. 122, 1-8 (2018).

156. Nieuwboer A, Kwakkel G, Rochester L et al. Cueing training in the home improves gait-related mobility in Parkinson's disease: the RESCUE trial. J. Neurol. Neurosurg. Psychiatry 78(2), 134-140 (2007).

-. Shows that cueing training may be a useful therapeutic adjunct to the overall management of gait disturbance in Parkinson's disease.

157. van den Heuvel MRC, Daffertshofer A, Beek PJ, Kwakkel G, van Wegen EEH. The effects of visual feedback during a rhythmic weight-shifting task in patients with Parkinson's disease. Gait Posture 48, 140-145 (2016).

158. Behrman AL, Teitelbaum P, Cauraugh JH. Verbal instructional sets to normalise the temporal and spatial gait variables in Parkinson's disease. J. Neurol. Neurosurg. Psychiatry 65(4), 580-582 (1998).

159. Tamir R, Dickstein R, Huberman M. Integration of motor imagery and physical practice in group treatment applied to subjects with Parkinson's disease. Neurorehabil. Neural Rep. 21(1), 68-75 (2007).

160. Abbruzzese G, Avanzino L, Marchese R, Pelosin E. Action observation and motor imagery: innovative cognitive tools in the rehabilitation of Parkinson's Disease. Parkinsons Dis. 2015, 124214 (2015).

161. Nanhoe-Mahabier W, Allum JH, Pasman EP, Overeem S, Bloem BR. The effects of vibrotactile biofeedback training on trunk sway in Parkinson's disease patients. Parkinsonism Relat. Disord. 18(9), 1017-1121 (2012).

162. Volpe D, Giantin MG, Maestri R, Frazzitta G. Comparing the effects of hydrotherapy and land-based therapy on balance in patients with Parkinson's disease: a randomized controlled pilot study. Clin. Rehabil. 28(12), 1210-1217 (2014).

163. Clerici I, Maestri R, Bonetti F et al. Land plus aquatic therapy versus land-based rehabilitation alone for the treatment of freezing of gait in Parkinson disease: a randomized controlled trial. Phys. Ther. 99(5), 591-600 (2019).

164. Volpe D, Signorini M, Marchetto A, Lynch T, Morris ME. A comparison of Irish set dancing and exercises for people with Parkinson's disease: a Phase II feasibility study. BMC Geriatr. 13, 54 (2013).

165. Shanahan J, Morris ME, Bhriain ON, Volpe D, Lynch T, Clifford AM. Dancing for Parkinson disease: a randomized trial of irish set dancing compared with usual care. Arch. Phys. Med. Rehabil. 98(9), 1744-1751 (2017).

166. Raglio A. Music therapy interventions in Parkinson's disease: the state-of-the-art. Front. Neurol. 6, 185 (2015).

167. Giladi N, Manor Y, Hilel A, Gurevich T. Interdisciplinary teamwork for the treatment of people with Parkinson's disease and their families. Curr. Neurol. Neurosci. Rep. 14(11), 493 (2014).

168. Post B, van der Eijk M, Munneke M, Bloem BR. Multidisciplinary care for Parkinson's disease: not if, but how! Postgrad. Med. J. 87(1031), 575-578 (2011).

169. Rochester L, Espay AJ. Multidisciplinary rehabilitation in Parkinson's disease: a milestone with future challenges. Mov. Disord. 30(8), 1011-1013 (2015).

170. van der Marck MA, Bloem BR, Borm GF, Overeem S, Munneke M, Guttman M. Effectiveness of multidisciplinary care for Parkinson's disease: a randomized, controlled trial. Mov. Disord. 28(5), 605-611 (2013).

171. Marumoto K, Yokoyama K, Inoue T et al. Inpatient enhanced multidisciplinary care effects on the quality of life for Parkinson disease: a quasi-randomized controlled trial. J. Geriatr. Psychiatry Neurol. 32(4), 186-194 (2019).

172. Dodel RC, Singer M, Köhne-Volland R et al. The economic impact of Parkinson's disease. An estimation based on a 3-month prospective analysis. Pharmacoeconomics 14(3), 299-312 (1998).

173. Martinez-Martin P, Macaulay D, Jalundhwala YJ et al. The long-term direct and indirect economic burden among Parkinson's disease caregivers in the United States. Mov. Disord. 34(2), 236-245 (2019).

174. Suh DC, Pahwa R, Mallya U. Treatment patterns and associated costs with Parkinson's disease levodopa induced dyskinesia. J. Neurol. Sci. 319, 24-31 (2012).

175. Winter Y, von Campenhausen S, Reese JP et al. Costs of Parkinson's disease and antiparkinsonian pharmacotherapy: an Italian cohort study. Neurodegener. Dis. 7(6), 365-372 (2010).

176. Gage H, Kaye J, Owen C, Trend P, Wade D. Evaluating rehabilitation using cost-consequences analysis: an example in Parkinson's disease. Clin. Rehabil. 20(3), 232-238 (2006).

177. Ypinga JHL, de Vries NM, Boonen LHHM et al. Effectiveness and costs of specialised physiotherapy given via ParkinsonNet: a retrospective analysis of medical claims data. Lancet Neurol. 17(2), 153-161 (2018).

178. Bloem BR, Munneke M. Revolutionising management of chronic disease: the ParkinsonNet approach. BMJ 348, g1838 (2014).

-. Offers evidence that specialized and net-organized physiotherapy is associated with fewer PD-related complications and lower costs in real-world practice. 